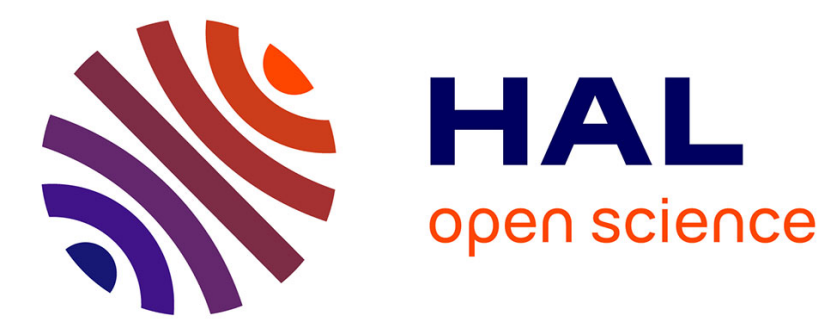

\title{
Le gros chêne m'a dit
}

Louis Lanier

\section{To cite this version:}

Louis Lanier. Le gros chêne m'a dit. Revue forestière française, 2005, 57 (6), pp.541-550. $10.4267 / 2042 / 5006$. hal-03449495

\section{HAL Id: hal-03449495 \\ https://hal.science/hal-03449495}

Submitted on 25 Nov 2021

HAL is a multi-disciplinary open access archive for the deposit and dissemination of scientific research documents, whether they are published or not. The documents may come from teaching and research institutions in France or abroad, or from public or private research centers.
L'archive ouverte pluridisciplinaire HAL, est destinée au dépôt et à la diffusion de documents scientifiques de niveau recherche, publiés ou non, émanant des établissements d'enseignement et de recherche français ou étrangers, des laboratoires publics ou privés. 


\section{HISTOIRE ET TERAITOIRES}

\section{Le Gros Chêne M’A dit}

\section{LOUIS LANIER}

Dans l'article qui suit, Louis Lanier fait parler un gros chêne d'une forêt privée de Haute-Saône ; c'est en fait l'occasion d'une réflexion sur deux siècles et demi d'évolution d'un peuplement issu du taillis-sous-futaie et sur l'orientation à donner à cette forêt dans le troisième âge du gros chêne.

Personnellement, je perçois un enseignement et un débat à la lecture de l'article :

L'enseignement : le mode de gestion par le groupement, continu et stable depuis maintenant plus de cinquante années, permet l'application de la méthode d'aménagement du contrôle qui permet elle-même un suivi précis et une adaptation permanente à l'évolution du peuplement parcelle par parcelle. C'est éminemment précieux mais c'est consommateur de temps et nécessite des compétences sylvicoles affirmées, cela n'est donc pas possible dans toutes les forêts ; voilà pourquoi il a paru utile de publier cet article dans la Revue forestière française.

Le débat : la méthode du contrôle est un outil qui éclaire un choix. Ce choix appartient au propriétaire, au cas présent le conseil de gérance du groupement forestier, et il est relatif à un état de fait : la dynamique des essences conduit à une augmentation de la place du Hêtre et du Sapin au détriment du Chêne. Faut-il laisser faire, voire encourager, cette évolution au motif, comme le dit le Gros Chêne au préjudice de ses jeunes confrères, qu'ils sont facilement gélifs ou, au contraire, la combattre au motif que la valeur économique du Hêtre est capricieuse et souvent décevante et que le Chêne, dans la perspective du changement climatique, sera autant, et peut-être davantage, dans son aire optimale que le Hêtre et le Sapin ?

Voilà une interrogation qui devrait intéresser bien des lecteurs de la Revue.

Dominique DANGUY des DÉSERTS(*)

Notre lointain collègue et prédécesseur Jean ${ }^{(1)}$ faisait parler les animaux pour mieux comprendre les hommes. Ne reculant devant aucune barrière biologique et tenant compte du fait que mon humour ne peut plus, en aucun cas, nuire à mon avancement, j'oserai faire parler les arbres ou plutôt un arbre, le Gros Chêne du Poirmont. Voici ce qu’il m'a dit :

Étant né très précisément en 1754, je viens de dépasser les deux siècles et demi, ce qui n'a rien d'extraordinaire pour un Chêne, mais mon histoire, elle, mérite certainement d'être contée. J'y ajouterai un peu de géographie pour faire bonne mesure.

1754, c'est aux confins de plusieurs États aux contours incertains que j'ai vu le jour, dans une contrée où une goutte d'eau tombée du ciel - elles sont nombreuses - finira sa course dans

(*) Conseil général du Génie rural, des Eaux et des Forêts - 140 bis, rue de Rennes - F-75006 PARIS.

(1) Jean de La Fontaine (1621-1695), le célèbre écrivain français, fut Maître des Eaux et Forêts de 1652 à 1672 à Château-Thierry. 
les mers très éloignées et diverses selon l'endroit précis où elle a chu. Si elle est tombée près de chez moi, qui suis dans le vaste bassin versant de la Saône, elle rejoindra le Rhône et plein sud se retrouvera en Méditerranée. Si elle est tombée plus au nord, dans les Vosges toutes proches, elle descendra la Moselle et par le Rhin sera absorbée par la Mer du Nord. Si, enfin, elle tombe un peu plus à l'ouest, près de la Haute-Marne, c'est vers la Seine, Paris et la Manche qu'elle s'écoulera. 1754, c'est encore une période où l'histoire de nos régions et même leurs frontières ne sont pas encore fixées. Ce n'est que douze ans après ma naissance que la Lorraine sera rattachée à la France. L'Empire romain germanique a éclaté en multiples principautés, qui cherchent à s'étendre vers l'ouest. Quant à la Franche-Comté, elle a récupéré une certaine autonomie, après les envahissements par les Français au XVII siècle.

Bref, j'aurais vécu assez tranquille au fond de mes forêts, peu accessibles, plus ou moins sauvages, s'il n'y avait pas, de plus en plus actifs dans les régions, les industriels du fer, du verre et, évidemment, du bois qui s'intéressaient tous à nous, nous qui fournissions seuls et en abondance la source d'énergie nécessaire au chauffage des habitants bien sûr, mais aussi aux exigeants industriels. Le sort des vastes forêts feuillues de la région était donc scellé. Le traitement prioritaire était le taillis, avec des révolutions de plus en plus courtes, avec quelques réserves en taillis-sous-futaie, en vue de régénérer Chênes, Charmes et Hêtres lorsque les souches des précédents taillis étaient épuisées.

Mais avant de vous raconter ma vie tout au long des deux derniers siècles, laissez-moi vous dire quel miracle fut ma naissance : je dois l'existence à la chance bien sûr, car le gland que je fus avant de devenir petit chêne tomba, en fin d'octobre 1753, lors d'une glandée d'une exceptionnelle profusion, entre deux turricules ${ }^{(2)}$ de vers de terre. Je ne remercierai jamais assez les lombrics qui les avaient érigés, car une glandée profuse signifiait, pour les paysans locaux, l'autorisation de panage des porcs, ce qui ajoutait à la gourmandise habituelle des nombreux sangliers le risque de voir nos glands pleins d'avenir se transformer en jambons fumés.

Bref, j'eus la chance d'être piétiné par un pied de porc et enfoncé de quelques centimètres entre mes deux turricules protecteurs. Le printemps 1754 fut très doux, bien arrosé comme à l'habitude et j'eus le temps d'enfoncer ma radicule dans le sol ameubli par les lombrics des quarante bons centimètres me permettant de toujours trouver l'humidité nécessaire à la bonne croissance de ma minuscule tigelle. Une fois mes cotylédons épuisés de leurs réserves, ma tigelle grimpa dès ma première année d'une bonne quinzaine de centimètres sous la protection d'un roncier providentiel. En effet, dans l'hiver 1755-1756, les chevreuils préférèrent plusieurs fois les feuilles de mon roncier à mes maigres bourgeons, pourtant bien aoûtés. Ils préféraient encore aux ronces les rejets de fruitiers forestiers (Merisiers, Alisiers, Pommiers et Poiriers sauvages, encore assez nombreux à cette époque...). J'atteignis rapidement une taille suffisante pour me dresser, tel un fier baliveau au milieu de quelques congénères et de rejets d'essences diverses. J'échappai encore, au tournant du XIX siècle, aux feux de camp des armées napoléoniennes, qui passaient fréquemment dans la région.

Mais c'est à partir de 1813 que mes craintes furent les plus vives : en effet, je fus soumis par la volonté de mes propriétaires, maîtres de forges de la région (vallées de la Semouse et de l'Augronne principalement), à un régime de taillis-sous-futaie à révolution de 25 ans. C'est ainsi que, dans le massif dit du Bas-Poirmont où je me dressais fièrement comme un jeune chêne adolescent de soixante ans, je ne dus ma survie qu'à ma forme caractéristique en pommier avec

(2) Dans une bonne partie de la Haute-Saône proche des Vosges, sur des stations où les grès intermédiaires du Trias sont souvent accompagnés de limons, une variété de lombrics géants assurent le drainage vertical en creusant des galeries de plus de 6 mm de diamètre. Ils remontent en surface en constituant des turricules atteignant presque les dimensions d'une taupinière. À noter accessoirement le danger constitué par ces “taupinières lombriciennes” pour les marteleurs qui, les yeux en l'air pour juger la qualité des arbres, se tordent souvent les pieds sur les turricules gelés. 
un tronc court mais bien droit et bien cylindrique, une première branche latérale vers 6 à 8 mètres de haut et surtout un large houppier garant de glandées abondantes. Associé à deux autres massifs de même surface, nous réussissions à produire chaque année sur une coupe de 31 hectares, associée pour ce qui concerne le Bas-Poirmont à une coupe ou deux de 8 hectares, la charbonnette et le charbon nécessaires aux forges.

Ceci signifiait que le Bas-Poirmont avec ses 261 hectares pouvait fournir huit ans de production, les deux autres massifs en produisant chacun autant : 24 ans de production étaient ainsi assurés, les besoins de la vingt-cinquième année étant satisfaits par quatre coupes supplémentaires de 8 à 10 hectares.

Il n'est donc pas étonnant de retrouver, dans le parcellaire actuel, des parcelles qui, côte à côte, totalisent 31 hectares, ainsi que quelques parcelles, faciles d'accès, faisant 8 à 10 hectares. Et ce régime dura jusqu'à la Grande Guerre (1914-1918), en fait tant que le charbon de terre n'eut pas remplacé le charbon de bois pour alimenter les forges en énergie.

L’École royale forestière de Nancy et son directeur Lorentz eurent beau déplorer que "le régime $d u$ taillis (sous-futaie) a maintenu les forestiers dans l'enfance... ", il était tellement commode à appliquer et le lobby industriel si puissant que les conversions préconisées par les forestiers français formés à Nancy furent ralenties, certaines même inversées...

Pour ce qui me concernait, le régime du taillis-sous-futaie, qui continua donc à être appliqué jusqu'après la Grande Guerre, n'était pas trop dangereux dans la mesure où je fus régulièrement conservé lors des balivages successifs.

C'est vers la fin du XIXe siècle qu'un tout autre rôle me fut dévolu. En effet, un artisan maçon de la région, nommé Barrault (nom assez commun dans l'Est), en vint à souffrir de la vue au point de craindre la cécité. Les médecins de l'époque n'étaient guère en mesure de soigner ces troubles, qu'il s'agisse de glaucome, de cataracte, de dégénérescence rétinienne ou des conséquences d'un accident vasculaire cérébral. Aussi Barrault s'en remit-il à une indulgence céleste et fit-il le vœu de bâtir de ses mains une chapelle dédiée à la Vierge, dans l'espoir que sa vue lui restât. Ainsi fut bâtie la chapelle qui porte son nom, à l'ombre tutélaire d'une de mes grosses branches et en bordure d'un chemin de grande randonnée. Ce lieu de rendez-vous figure sur les cartes et ma grosse branche indique la direction du sud-ouest pour les éventuels pèlerins, sur le chemin de la Grotte de Lourdes et de Saint-Jacques-de-Compostelle.

Venons-en maintenant à la période plus moderne qui, pour moi et pour le massif du BasPoirmont, constitue un changement radical dans les orientations de la production forestière.

Le bois de feu étant de moins en moins employé, aussi bien dans l'industrie que dans les foyers domestiques ${ }^{(3)}$, c'est tout naturellement vers la production de bois d'œuvre que se sont orientés les gestionnaires, donc vers la conversion progressive en futaie mélangée.

De 1927 à 1952, le gestionnaire profitant des rejets de souches, de Chêne bien sûr, mais aussi, curieusement, de nombreux Hêtres ${ }^{(4)}$, opéra un balivage intensif des meilleurs sujets, réservant en moyenne à l'hectare 437 baliveaux, modernes et anciens des meilleures espèces : 233 Hêtres, 170 Chênes et 34 divers (Bouleaux, Aulnes, Frênes, ces derniers très rares et pratiquement ni Érables ni fruitiers).

(3) Avec une notable exception durant les années sombres de 1940 à 1945 (avec l'usage des gazogènes...) et avec peut-être un avenir qui semble se profiler aujourd'hui.

(4) Dont on retrouve encore la trace de nos jours : des gros Hêtres sont souvent bifides, voire trifides à la base, alors qu'on s'attendrait plutôt à n'avoir que des Hêtres de franc pied. Le Hêtre, habituellement, rejette bien de souche en altitude (Vosges, Jura, Pyrénées) et très mal sinon pas du tout à l'altitude du Poirmont $(400 \mathrm{~m})$. 
Par ailleurs et afin d'enrichir plus rapidement les peuplements plus ou moins ruinés par plusieurs siècles de traitement en taillis, on procéda à la plantation entre 1926 et 1950 de 380000 Sapins, à raison de 1450 plants par hectare, en plein dans les parcelles 11 et 12, qui venaient d'être parcourues par des coupes, et par bouquets de 1 à 3 ares dans les autres parcelles.

Ces Sapins sont aujourd'hui de jeunes adultes et font l'objet d'éclaircies et, pour les plus jeunes ou au sein des bouquets de régénération qu'ils ont déjà constitués, d'élagages d'individus sélectionnés.

\section{CONSTITUTION DU GROUPEMENT FORESTIER}

Un changement radical est intervenu dans la vie du massif du Bas-Poirmont en 1953, c'est-à-dire juste avant mon bi-centenaire, lorsque fut constituée la "Société civile forestière et de Reboisement du Poirmont".

Composée au départ (22 janvier 1953) de 72 membres, parmi lesquels quelques forestiers ayant servi ou encore en activité, dans l'Est de la France, ainsi que des parents ou connaissances d'industriels locaux, la Société a adopté, trois ans plus tard, le 17 novembre 1956 le statut de Groupement forestier. Celui-ci est, encore de nos jours, constitué de 62 porteurs, se partageant les 235 parts initiales (de $2000 \mathrm{~F}$ à l'origine, estimées aujourd'hui à $2000 €$ ). Les statuts viennent d'en être révisés (12 décembre 2001) pour tenir compte de l'évolution de la législation concernant ces groupements.

\section{LA GESTION DE 1953 À NOS JOURS}

Le Gros Chêne m'a encore dit :

Ah ! J'en ai vu passer des têtes galonnées et même celle, étoilée, d'un célèbre forestier connu sous le surnom de "Mimile" (5). Père de la coupe d'abri, il a su mettre à profit et surtout diffuser dans toute l'aire potentielle de son élève préféré, le Sapin, sa méthode issue de cette observation selon laquelle le Sapin est une des rares essences qui peuvent subsister, végéter diront certains, de longues années à l'ombre d'autres arbres, feuillus ou résineux et se remettre à pousser vigoureusement dès que l'espace nécessaire lui sera offert. Certains forestiers suisses prétendent même que le fait de rester longtemps en couveuse sous abri confèrerait à ces sapins, de moins de 40 ans par exemple, des qualités particulières : en effet, ils disposent d'un système racinaire disproportionné par rapport à leur partie érigée et, dès lors, auraient une croissance plus vigoureuse que les sapinots de même taille, mais plus jeunes. Ce que l'on doit seulement retenir, c'est que des hêtraies adultes, par exemple celles de la forêt de Tannières près d'Épinal, se sont transformées en quelques décennies en hêtraies sous-étagées de sapins, des semenciers de cette dernière essence vivant à proximité. Ce sont maintenant des sapinières-hêtraies, qui peuvent évoluer, au gré des sylviculteurs, vers l'enrichissement en résineux ou, plus prudemment, vers des mélanges Hêtre et Sapin.

\section{Histoire récente du Poirmont}

Les opérations d'enrichissement des peuplements se sont succédé durant la période 1962-1972 ainsi que l'atteste la comparaison entre les inventaires en plein de 1962 et de dix ans plus tard.

(5) Émile Lachaussée (1899-1966), qui fut notamment membre du Comité consultatif des Aménagements à la Direction générale des Eaux et Forêts. 
Dès 1953, l'objectif fixé à moyen-long terme était une hêtraie-sapinière en éliminant progressivement les Chênes jugés trop gélifs.

La comparaison d'inventaires permettait d'estimer la production annuelle globale des 261 ha du massif à $1122 \mathrm{~m}^{3}$, dont il était prélevé $850 \mathrm{~m}^{3} / \mathrm{an}$, soit environ les trois quarts. Ici et dans tout ce qui suit, il s'agit de volume aménagement, calculé au moyen d'un tarif établi pour le Poirmont à partir d'une analyse de tiges. Ce tarif a servi pour chacun des inventaires depuis celui de 1962.

Le plan de gestion de 1973, établi pour 13 ans (1973-1985), prévoyait une conversion des peuplements enrichis et plus ou moins mûrs, avec un quartier de régénération de 48 ha, un autre de préparation sur 57 ha supplémentaires, le reliquat, soit moins de 160 ha devant être parcouru par des coupes prudentes, sanitaires pour l'essentiel, en vue d'une conversion plus lointaine, ou par des coupes de régénération ponctuelle, par bouquets ou parquets, de quelques ares à un hectare.

Ces données montrent bien toutes les difficultés qu'ont connues les gestionnaires d'anciens taillissous-futaie pour établir, même à long terme, des surfaces sensiblement équilibrées entre les différentes classes d'âge. Il s'y ajoutait au Poirmont la difficulté supplémentaire de disposer comme essences principales du Chêne rouvre, du Hêtre et du Sapin dont les âges d'exploitabilité diffèrent notablement. Quelques rares plantations de Douglas dans les vides laissés par des échecs de régénération étaient censées "amortir" les déséquilibres entre peuplements principaux, en songeant à d'éventuels relais de production.

Le deuxième plan simple de gestion (PSG), établi pour 10 ans (1986-1995), proposait de convertir en futaie pleine par un groupe de parcelles (ou de parties de parcelles) constituant un quartier de régénération de 71 hectares c'est-à-dire plus du

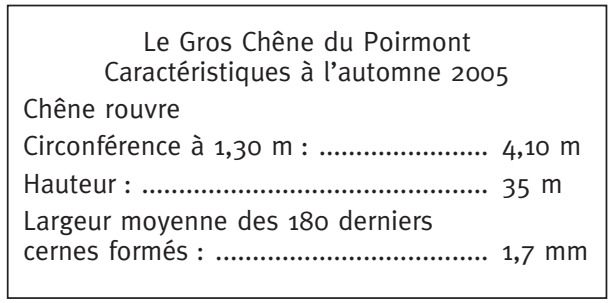

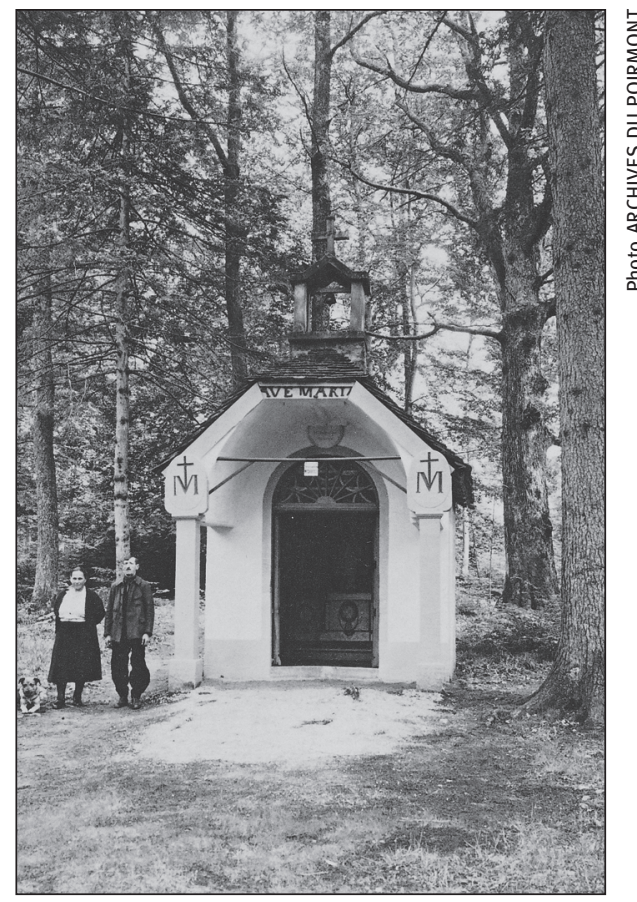

Forêt du Bas-Poirmont (Haute-Saône). Vieux chêne vers 1953 (en haut) et en 2005 (en bas)

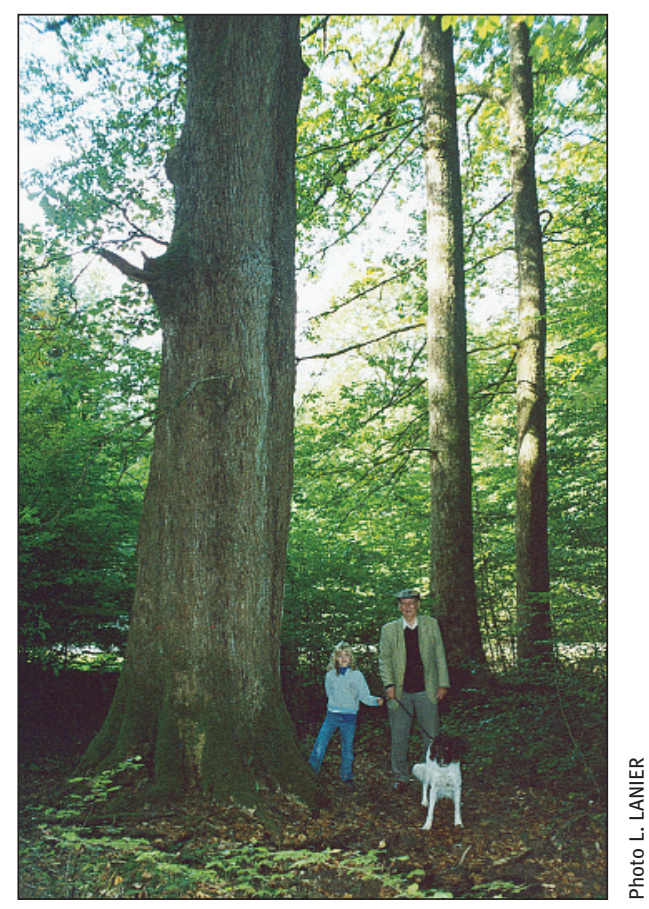


POIRMONT - Inventaire Juillet-Août 1972 Précomptables 20 et +

\begin{tabular}{|c|c|c|c|c|c|c|c|}
\hline \multirow{2}{*}{ Parcelle } & \multirow{2}{*}{ Surface/ha } & \multicolumn{2}{|c|}{ Chêne } & \multicolumn{2}{|c|}{ Hêtres et divers feuillus } & \multicolumn{2}{|c|}{ Résineux } \\
\hline & & Nombre & Volume & Nombre & Volume & Nombre & Volume \\
\hline $1 \ldots \ldots \ldots \ldots \ldots$ & 9,72 & 1011 & 617,3 & 607 & 544,3 & 7 & 1,6 \\
\hline $2 \ldots \ldots \ldots \ldots \ldots$ & 12,48 & 1408 & 796,8 & 513 & 312,4 & 202 & 44,9 \\
\hline $3 \ldots \ldots \ldots \ldots$ & 9,38 & 778 & 528,9 & 257 & 159,7 & 149 & 39,6 \\
\hline $4 \ldots \ldots \ldots \ldots \ldots$ & 9,06 & 766 & 630,3 & 386 & 296,2 & 143 & 51,4 \\
\hline $5 \ldots \ldots \ldots \ldots \ldots$ & 12,51 & 876 & 621,1 & 1219 & 698,8 & 194 & 69,0 \\
\hline $6 \ldots \ldots \ldots \ldots \ldots$ & 16,93 & 1534 & 1042,4 & 2089 & 1292,0 & 557 & 270,6 \\
\hline $7 \ldots \ldots \ldots \ldots$ & 15,58 & 1569 & 1096,6 & 2418 & 1287,8 & 4 & 0,8 \\
\hline $8 \ldots \ldots \ldots \ldots \ldots$ & 10,88 & 893 & 832,9 & 971 & 504,9 & 8 & 1,8 \\
\hline $9 \ldots \ldots \ldots \ldots \ldots$ & 10,93 & 1161 & 790,3 & 1214 & 708,4 & 6 & 1,2 \\
\hline $10 \ldots \ldots \ldots \ldots \ldots \ldots$ & 10,24 & 882 & 625,2 & 1388 & 962,9 & 4 & 1,6 \\
\hline $11 \ldots \ldots \ldots \ldots \ldots$ & 15,23 & 707 & 385,5 & 370 & 193,2 & 12 & 2,6 \\
\hline $12 \ldots \ldots \ldots \ldots \ldots \ldots$ & 16,32 & 3030 & 1676,7 & 1506 & 876,7 & 21 & 5,7 \\
\hline $13 \ldots \ldots \ldots \ldots \ldots$ & 17,40 & 1884 & 1490,6 & 2159 & 1919,0 & - & - \\
\hline $14 \ldots \ldots \ldots \ldots \ldots$ & 10,39 & 743 & 529,6 & 1702 & 1236,6 & 2 & 0,4 \\
\hline $15 \ldots \ldots \ldots \ldots \ldots$ & 12,67 & 1081 & 918,3 & 1589 & 908,5 & 38 & 8,5 \\
\hline $16 \ldots \ldots \ldots \ldots \ldots$ & 17,97 & 1536 & 1188,0 & 1432 & 974,4 & 7 & 1,4 \\
\hline $17 \ldots \ldots \ldots \ldots \ldots$ & 14,16 & 1312 & 1010,0 & 1679 & 1029,9 & 26 & 6,4 \\
\hline $18 \ldots \ldots \ldots \ldots \ldots \ldots$ & 15,88 & 1099 & 766,6 & 1329 & 849,9 & 6 & 1,4 \\
\hline $19 \ldots \ldots \ldots \ldots \ldots$ & 15,62 & 961 & 1048,8 & 1239 & 842,8 & 17 & 4,1 \\
\hline $20 \ldots \ldots \ldots \ldots \ldots$ & 8,21 & 705 & 769,6 & 625 & 578,0 & - & - \\
\hline TOTAUX & 261,56 ha & 23936 & 17360,5 & 24692 & 1676,4 & 1403 & 513,0 \\
\hline
\end{tabular}

quart de la surface totale du massif, avec comme proportion des essences objectifs : Hêtre $60 \%$, résineux $25 \%$ et Chêne $15 \%$.

C'est dans les années 1990 qu'en tant que vieux chêne, lieu de rendez-vous pour l'étude de la forêt, j'ai vu passer les élèves de Nancy et leurs enseignants. Tous se sont accordés pour trouver l'enrichissement de la forêt, depuis les cinquante dernières années, remarquable... au point de nécessiter en maints endroits des éclaircies vigoureuses. Encore fallait-il que le PSG de $3^{\text {e }}$ génération, s'appuyant sur des données phytosociologiques récentes ainsi que sur une cartographie des peuplements détaillée, fournisse des indications chiffrées sur le prélèvement, aussi précises que possible, ainsi qu'un tableau des passages en coupe (passés et futurs).

À la lecture, en 1991, d'un article de Pardé(6), notant entre autres avantages l'universalité potentielle de la méthode du contrôle s'appuyant sur des comparaisons d'inventaires, il a été bâti un PSG qui retient les trois principales composantes d'une idée, exprimée pour la première fois il y

(6) PARDÉ (J.). - La Méthode du contrôle, d'hier à aujourd'hui. - Revue forestière française, vol. XLIII, n 3, 1991, pp. 185-201. 


\begin{tabular}{|c|c|c|c|}
\hline \multicolumn{2}{|c|}{ Total } & \multirow{2}{*}{$\begin{array}{c}\text { Volume } \\
\text { arbre moyen }\end{array}$} & \multirow{2}{*}{ Volume/ha } \\
\hline Nombre & Volume & & \\
\hline 1625 & 1163,2 & 0,716 & 126,16 \\
\hline 2123 & 1154,1 & 0,544 & 92,48 \\
\hline 1184 & 728,2 & 0,615 & 77,63 \\
\hline 1295 & 977,9 & 0,755 & 107,94 \\
\hline 2289 & 1388,9 & 0,607 & 111,02 \\
\hline 4180 & 2605,0 & 0,623 & 153,87 \\
\hline 3991 & 2385,2 & 0,598 & 153,09 \\
\hline 1872 & 1339,6 & 0,716 & 123,12 \\
\hline 2381 & 1499,9 & 0,630 & 137,23 \\
\hline 2274 & 1589,7 & 0,699 & 155,24 \\
\hline 1089 & 581,3 & 0,534 & 38,04 \\
\hline 4557 & 2559,1 & 0,562 & 156,81 \\
\hline 4043 & 3409,6 & 0,843 & 195,95 \\
\hline 2447 & 1766,6 & 0,722 & 170,03 \\
\hline 2708 & 1835,3 & 0,678 & 144,25 \\
\hline 2975 & 2163,8 & 0,727 & 120,41 \\
\hline 3017 & 2046,3 & 0,678 & 144,51 \\
\hline 2434 & 1617,9 & 0,665 & 101,88 \\
\hline 2216 & 1890,7 & 0,853 & 121,04 \\
\hline \multirow[t]{2}{*}{1330} & 1347,6 & 1,013 & 164,14 \\
\hline & & Moyenne & Moyenne \\
\hline 50031 & 34049,9 & 0,681 & 130,40 \\
\hline
\end{tabular}

a près d'un siècle et demi, alors que j'étais un jeune chêne de 120 ans, l'âge actuel de ma progéniture qui m'entoure.

La sylviculture se conduit par parcelles entières et indépendantes. L'unité de la forêt ne joue qu'un rôle secondaire. Ceci est d'autant plus important en forêt privée que les groupements forestiers comme le nôtre peuvent s'agrandir par acquisition de boisements à améliorer (article 2 des statuts) et sont régulièrement l'objet de mutations qui se résolvent par la vente de "parts". Chaque part, pour le massif principal du Poirmont, représentait lors de sa constitution en 1953 exactement 1,10 hectare (235 parts pour 261 hectares).

Par le jeu des acquisitions plus récentes, un petit massif résineux d'environ 8 hectares sur la Route des Crêtes à la Montagne, une forêt d'environ 25 hectares, mélangée feuillus-résineux près de Bains-les-Bains, enfin tout récemment, un massif de près de 50 hectares au sud de Luxeuil, l'équivalent en surface théorique d'une part atteint près d'un hectare et demi.

La gestion par la méthode du contrôle ne s'exerce que sur le massif principal, mais rien n'empêcherait dans l'avenir d'étendre la méthode aux autres boisements, pourvu qu'un parcellaire constant y soit établi. Au Poirmont, sur nos parcelles d'une surface moyenne de 13 hectares, les rotations sont courtes (de 7 à 10 ans) selon l'état réel de chaque parcelle et, en particulier, l'étendue des plages de régénération. Des passages à mi-rotation sont même prévus à la demande pour les parcelles en grande partie (parcelle 20) ou totalement régénérées (parcelle 8), afin d'y pratiquer les cloisonnements, dégagements, dépressages, puis nettoiements nécessaires.

Voyons plus en détail comment se réalisent les comparaisons d’inventaires. L'inventaire de référence est celui de 1972 dont les résultats figurent au tableau I (ci-contre). Les inventaires récents figurent au tableau II (p. 548).

Prenons par exemple la parcelle 17, qui vient en tour en 2005 et qui a été, comme le seront quelques autres parcelles avant 2010, terme de l'actuel PSG, inventoriée 3 fois depuis 1972. Ceci permet d'intéressants recoupements.

En 1972, la parcelle 17 (14 ha 16) comportait 1312 Chênes pour un volume de $1010 \mathrm{~m}^{3}$, 1679 Hêtres et feuillus divers (Charmes et Trembles pour la plupart et peu nombreux) pour un volume de $1029,9 \mathrm{~m}^{3}$, s'y ajoutaient 26 résineux précomptables pour 6,4 $\mathrm{m}^{3}$. Au total, la parcelle hébergeait 3017 tiges de plus de $15 \mathrm{~cm}$ de diamètre pour un volume de $2046,3 \mathrm{~m}^{3}$, soit $144 \mathrm{~m}^{3}$ par hectare. La tige moyenne mesurait donc environ deux tiers de mètre cube.

Ainsi qu'on peut le voir sur les figures 1 et 2 (p. 549) illustrant les deux derniers inventaires (1994 et 2004), la situation a bien évolué en un peu plus de 30 ans. 
TABLEAU II

Inventaires récents et analyses par parcelle

\begin{tabular}{|c|c|c|c|c|c|c|c|}
\hline Parcelle & S (ha) & $\begin{array}{c}\text { Date } \\
\text { inventaire(s) }\end{array}$ & Vol/ha $\left(\mathrm{m}^{3}\right)$ & N/ha & $\begin{array}{c}\Delta \mathrm{V} / \mathrm{ha} / \mathrm{an} \\
\left(\mathrm{m}^{3}\right)\end{array}$ & $\Delta \mathrm{V} / \mathrm{an}\left(\mathrm{m}^{3}\right)$ & $\mathrm{G} / \mathrm{ha}\left(\mathrm{m}^{2}\right)$ \\
\hline $1 \ldots \ldots \ldots$ & 9,22 & $1-1996$ & 231 & 195 & 6,58 & 60,7 & 20,3 \\
\hline $2 \ldots \ldots \ldots$ & 12,48 & 11 et $12-1994$ & 223 & 260 & 6,79 & 84,7 & 21,8 \\
\hline $3 \ldots \ldots \ldots$ & 9,38 & $11-2001$ & 198 & 231 & 7,4 & 69,4 & 19,1 \\
\hline $4 \ldots \ldots \ldots$ & 9,06 & $10-1996$ & 178 & 292 & 4,6 & 41,7 & 16,1 \\
\hline $5 \ldots \ldots \ldots$ & 12,51 & 9-1997 & 193 & 203 & 6,74 & 84,3 & 17,8 \\
\hline $6 \ldots \ldots \ldots$ & 16,93 & 11-1995 & 163 & 175 & 6,9 & 116,8 & 14,2 \\
\hline $7 \ldots \ldots \ldots$ & 15,58 & 9-1999 & 289 & 320 & 7,45 & 166 & 26,3 \\
\hline $8 \ldots \ldots \ldots \ldots$ & \multicolumn{7}{|c|}{ Non inventoriée, régénérée entre 1996 et 2003} \\
\hline $9 \ldots \ldots \ldots$ & 10,93 & $10-2001$ & 278 & 255 & 6,7 & 73,2 & 24,9 \\
\hline $10 \ldots \ldots \ldots \ldots$ & 10,24 & 9-1995 & 294 & 242 & 7,37 & 75,5 & 25,8 \\
\hline $11 \ldots \ldots \ldots$ & 15,28 & Hiver 1997-98 & $\begin{array}{l}\text { Estimé par } \\
1 \text { cloisonne- } \\
\text { ment sur } 10 \\
\text { à } 83\end{array}$ & $\begin{array}{c}\text { Élevé } \\
>1000 / \text { ha }\end{array}$ & \multicolumn{3}{|c|}{ Parcelle jeune en croissance } \\
\hline $12 \ldots \ldots \ldots$ & 16,38 & 10-1999 & 312 & 273 & 8,1 & 132,7 & 26,7 \\
\hline $13 \ldots \ldots \ldots \ldots$ & 17,40 & $10-2000$ & 369 & 217 & 7,7 & 134 & 29,75 \\
\hline $14 \ldots \ldots \ldots$ & 10,39 & 9-1993 & 288 & 280 & 7,16 & 74,4 & 24,2 \\
\hline $15 \ldots \ldots \ldots$ & 12,67 & 9-1994 & 255 & 250 & 7,5 & 95 & 23,4 \\
\hline $16 \ldots \ldots \ldots$ & 17,97 & $10-2003$ & 290 & 250 & 8,01 & 144 & 22,8 \\
\hline $17 \ldots \ldots \ldots$ & 14,16 & $11-1994$ & 257 & 244 & 5,84 & 82,7 & 23,2 \\
\hline $18 \ldots \ldots \ldots \ldots$ & 15,88 & $11-1993$ & 183 & 290 & 5,89 & 93,5 & 17,1 \\
\hline $19 \ldots \ldots \ldots$ & 15,62 & $11-1998$ & 193 & 270 & 5,92 & 92,5 & 16,4 \\
\hline $20 \ldots \ldots \ldots \ldots$ & 8,21 & 10-11-1995 & 120 & 140 & 5,6 & 46 & 11,5 \\
\hline
\end{tabular}

Après 22 ans en 1994, les Chênes sont un peu moins nombreux (1 196) pour un volume de $1510 \mathrm{~m}^{3}$. Ils ont donc bien prospéré. De même, les Hêtres et divers sont passés à 2096 pour un volume de $2089 \mathrm{~m}^{3}$, l'arbre moyen atteignant le mètre cube. Les résineux, enfin, se sont bien développés, même si beaucoup de Sapins sont encore petits. Le volume total pour la parcelle est de $3614 \mathrm{~m}^{3}$, soit $255 \mathrm{~m}^{3}$ par hectare. Les coupes et chablis, soigneusement relevés, ont ajouté $224 \mathrm{~m}^{3}$ au volume 1994 .

Si l'on retire du total $3614+224=3838 \mathrm{~m}^{3}$, le volume présent en 1972 , soit $2046,3 \mathrm{~m}^{3}$, on obtient l'accroissement $1786,7 \mathrm{~m}^{3}$ en 22 ans, soit $81,27 \mathrm{~m}^{3}$ par an.

La parcelle mesurant 14,16 ha, l'accroissement annuel par hectare est de 5,74 m3/ha/an, soit comparable à la moyenne des parcelles voisines, la parcelle 16 exceptée.

Entre 1994 et 2004, la parcelle a encore évolué, ainsi que le montre la comparaison entre les deux graphiques.

La disparition du Chêne paraît inéluctable, si des efforts importants ne sont pas consentis, lorsqu'une bonne glandée apparaît... Dégagements, dépressages, puis nettoiements seront néces- 


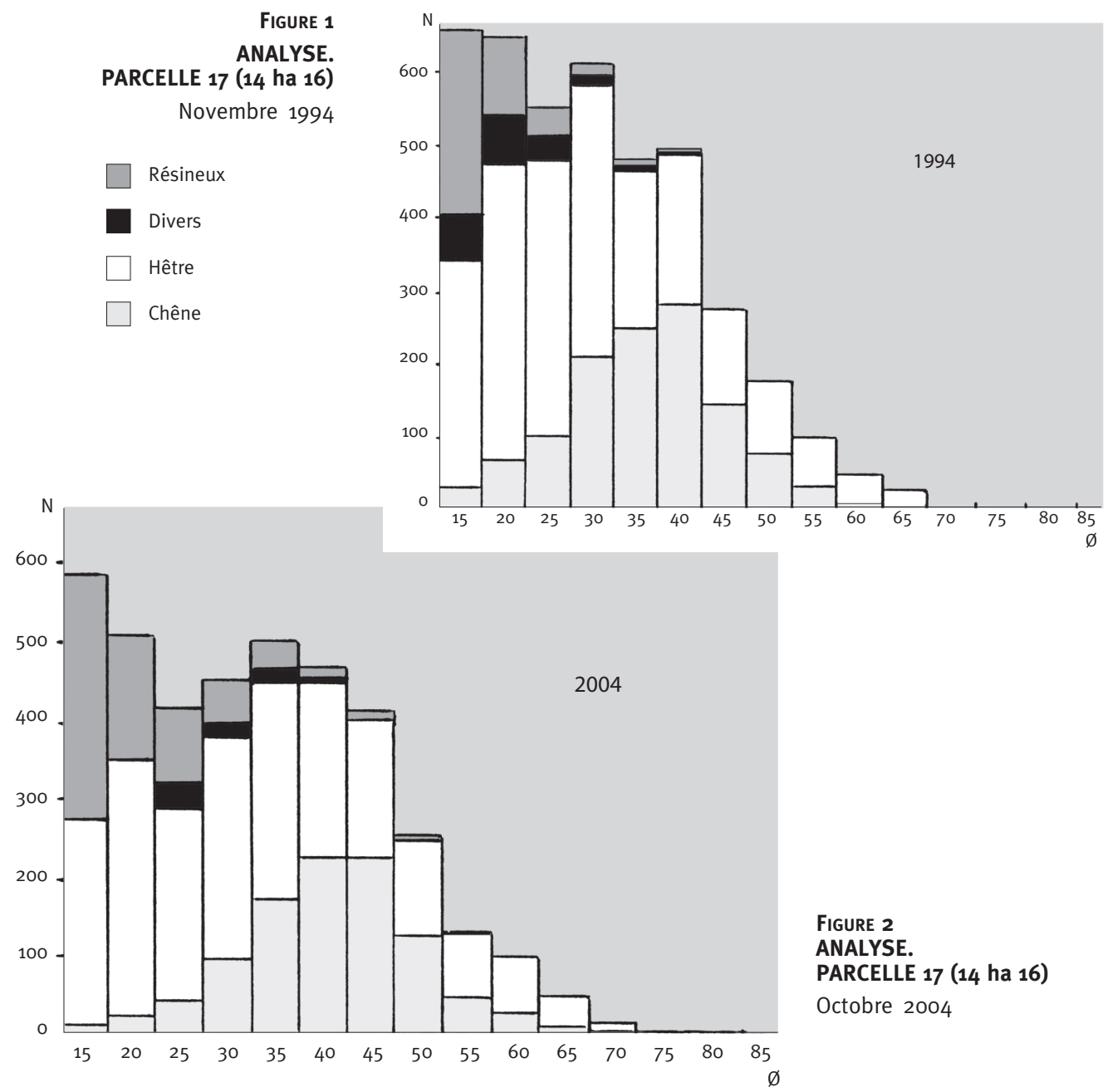

saires pour assurer un recrutement convenable, surtout dans les parcelles comme la 17 où la croissance des Chênes de 35 à $50 \mathrm{~cm}$ de diamètre est excellente pourvu qu'ils soient soustraits à la concurrence du Hêtre.

Le calcul de l'accroissement entre 1995 et 2004 se fait de la même manière :

Volume $2004+$ coupes de chablis 1995-2004 - volume 1994

$$
3941+669+41+3614=1037 \mathrm{~m}^{3} \text {, }
$$

en 10 ans, soit $103,7 \mathrm{~m}^{3}$ par année en moyenne.

La division par 14,16 hectares fournit l'accroissement annuel par hectare soit 7,32 m3/ha/an, ce qui est nettement meilleur que l'accroissement des vingt années précédentes.

Ce phénomène peu s'expliquer de diverses manières. Bien des comparaisons d'inventaires récents (Inventaire forestier national, placettes de recherche) ont montré que la plupart des essences dans la majorité des régions avaient tendance à s'accroître plus vite que par le passé. La sylvi- 
culture y est certainement pour quelque chose. Mais, dans le cas du Poirmont, nous notons que les parcelles où il y a le plus de résineux ont tendance à avoir des accroissements meilleurs. Il est également possible que les changements globaux de l'environnement conduisent à des fixations de carbone plus importantes sous forme de bois. Ce n'est sans doute pas pour rien que le label de certification PEFC a été accordé à notre groupement !

\section{PERSPECTIVES D'AVENIR ET CONCLUSIONS}

Reprenant la parole après toutes ces données chiffrées, le vieux chêne m’a confié :

Après la peur de ma vie, juste après la Noël de 1999, je me suis senti protégé, non seulement par mes enfants, qui me dépassent de nos jours par la taille, mais par tout le massif du Poirmont que j'ai vu évoluer depuis un demi-siècle. Ainsi, j'ai vu se développer, avec crainte au début, ces ambitieux de sapins qui ajoutaient rapidement leur concurrence à celle, déjà redoutable, des fayards nombreux dans le massif. Mais, durant les longs hivers, les Sapins restent bien verts, se décorent parfois d'un givre délicat, protègent la faune lorsque la neige est trop abondante. Une main anonyme a même, un jour, planté un petit Sapin bleu américain presque à mes pieds et je le regarde avec amitié en rêvant au Colorado.

Si, dans les années à venir, tout se passe comme mes propriétaires le souhaitent, c'est-à-dire si la crise économique, qui a frappé très fort le monde de la forêt après la tempête, finit par se dénouer, je pense que le prochain PSG (de quatrième génération déjà) respectera mon grand âge et me permettra peut-être d'envisager de poursuivre ma vie jusqu'en 2054 .

J'atteindrai alors l'âge respectable de 300 ans et si aucune maladie, aucun insecte et surtout aucune tempête nouvelle ne vient me faire mourir, je promets de faire tout mon possible pour voir pointer le vingt-deuxième siècle...

Louis LANIER

9, rue Bertrand Auerbach

F-54600 VILLERS-LES-NANCY

\section{LE GROS CHÊNE m'R DIT [Résumé]}

L'histoire du massif du Poirmont (Haute-Saône) est racontée par un arbre appelé “Le Gros Chêne”. Son récit, grâce à des éléments historiques et sylvicoles, permet de suivre l'évolution du massif et de sa gestion depuis la naissance de ce Gros Chêne en 1754 jusqu'à maintenant. Il est représentatif de maintes forêts de plaine du Nord-Est de la France.

\section{ШHAT THE TALL OAK TOLD mE [Abstract]}

The history of the Poirmont forest (Haute-Saône) as told by a tree called "The Tall Oak". The story uses historical and silvicultural elements to follow how the forest and its management developed from the inception of this Tall Oak in 1754 to date. The story is representative of how many forests in the plains of north-eastern France evolved. 